\title{
Rôle de la prosodie dans la construction de l'espace interdiscursif
}

\author{
Véronique Bourhis \\ IUFM de Bretagne - Université de Bretagne Occidentale \\ CREAD Rennes 2 - E.A.3875
}

\section{Introduction}

La prosodie désigne l'ensemble des traits suprasegmentaux. c'est-à-dire, dans le signal de parole, les modulations de la fréquence fondamentale (Fo), l'intensité, les durées et les pauses. Le terme inclut l'accentuation proprement dite, considérée comme le déclencheur de la structure intonative (Dell 1984).

Les fonctions de la prosodie dans le langage adulte se déclinent en 4 points. :

- une fonction structurale : la prosodie permet d'organiser la mise en perspective de l'information. Elle a un rôle démarcatif et est l'un des facteurs qui permet de distinguer le thème du rhème (Rossi et al 1981, Martin 1981, Fuchs et Le Goffic 1985).

- une fonction expressive en ce sens qu'elle traduit des phénomènes ectolinguistiques qui regroupent tout ce qui relève de la situation d'énonciation, de l'émotivité, de l'expressivité et de la personnalité du sujet (Fonagy 1983, Vroomen et al 1993, Gérard et Clément 1998).

- une fonction de contextualisation : elle permet d'identifier des items particuliers auxquels on peut attribuer une signification sémantique. Celle-ci dépend de l'identification des composantes pragmatiques de l'interaction, préalable à l'interprétation de l'énoncé. Elle permet également de prédire le poids informatif des unités associées aux stratégies énonciatives ce qui favorise la construction du message référentiel (Rossi 1999, Morel et Danon-Boileau 1992, Morel 1995).

- enfin la prosodie reflète l'intersubjectivité (Morel et Danon-Boileau 1998) : chaque indice qui entre en jeu dans l'intonation revêt, si on le prend isolément, une valeur de base et le couplage des indices permet de stabiliser les différentes fonctions de l'intonation. Ainsi, dans une interaction communicative, le locuteur fait en sorte que l'écouteur puisse saisir les éléments qu'il juge déterminants dans son discours. L'espace intersubjectif se construit en fonction des prédictions et des attentes supposées de l'écouteur par le locuteur et de l'attitude de l'écouteur.

Dans le cadre d'une linguistique appliquée à l'acquisition de la langue maternelle, la prosodie a fait l'objet de nombreuses études chez le bébé à partir de 6 mois (Crystal 1969,1986, Dore, 1975, Halliday 1975 pour l'anglais, Konopczynski 1986, 1990, 1991, 2000, pour le français), montrant la maîtrise progressive de contours prosodiques fonctionnels porteurs d'intention de communication. Toutes s'accordent à voir l'âge de 8 / 9 mois comme une période charnière : Konopczynski différencie le jasis, produit en solitaire, du protolangage en interaction, message intentionnel. Halliday signale pparition des « actes de signification ».

Cependant peu de linguistes se sont intéressés à l'âge entre deux et trois ans, la structuration prosodique étant supposé acquise. Au plan de l'acquisition l'enfant est entré entre 18 et 20 mois dans la phase d'explosion du vocabulaire: il possède 15 mots à 1 an, 200 à 2 ans, environ 530 à 3 ans (Bates et al 1995, Bassano 1998).

Au plan moteur, il coordonne ses mouvements : il marche, court, monte un escalier. Le « je » apparaît et avec lui la fonction symbolique (Morgenstern 1995).

Au plan prosodique, on admet que l'intonation rejoint celle de l'adulte : l'enfant exprime divers actes de langage et des modalités marqués intonativement, souvent de manière spécifique spécifique (Bernicot 1992, Bassano 1998).

La question se pose de savoir si les paramètres intonatifs sont stabilisés à cet âge ou si l'intonation joue un rôle spécifique dans le processus interdiscursif, et auquel cas, lequel ? 
Nous nous proposons de présenter dans cet article les principaux résultats de notre travail de thèse (Bourhis 2005), en essayant d'être le plus exhaustif et le plus précis possible.

\section{Hypothèse}

Selon Briand (1998), dans certaines situations, la focalisation attentionnelle, définie généralement comme l'orientation volontaire de l'attention sur des éléments centraux dans la réalisation d'une tâche, ne serait pas volontaire (endogène) mais involontaire (exogène). Il confirme ainsi qu'une partie de l'attention attribuée à des éléments périphériques peut être consommée par le traitement d'une informatrion incidente, information non directement liée à la tâche à accomplir (Cohen 1978).

Nous basant sur ces observations, nous posons l'hypothèse que, dans l'interaction dialogale, l'intonation est susceptible de favoriser la focalisation attentionnelle de l'enfant, en lui permettant de sélectionner dans des contextes précis différentes stratégies afin de parfaire ses intentions de communication et / ou de développer ses propres conduites discursives et plus largement développementales. En ce sens, elle aurait une fonction cognitive, en permettant de structurer non seulement non seulement les composantes d'une syntaxe balbutiante, mais aussi de caractériser certaines opérations cognitives spécifiques.

\section{Corpus et méthode}

\subsection{Corpus}

Le corpus est une monographie entre $2 ; 6$ et $3 ; 1$.

Afin de ne pas restreindre le champ des comportements langagiers, nous avons opté pour une méthode "naturelle" : celle du corpus spontané recueilli en milieu familial. Les échanges sont variés et leur contenu hétérogène. L'enfant, de sexe féminin, est la troisième et dernière de la fratrie, le père est médecin et la mère enseignante.

12 enregistrements ont été réalisés, dont 11 exploitables et 8 retenus pour le traitement des données (1 par mois), pour une durée de : $3 \mathrm{~h} 9 \mathrm{~min} 23 \mathrm{~s}$, et un total de 1217 interventions.

L'intérêt de l'étude d'un corpus unique réside dans le fait qu'elle donne une vision statique et globale d'une activité, et qu'elle permet notamment de développer des outils d'analyse spécifiques, de croiser des paramètres ponctuels : quelles sont les zones qui posent problème, quelles sont celles qui relèvent d'une problématique cognitive, pragmatique, syntaxique ? La monographie permet une analyse fine d'une situation précise et peut faire émerger des tendances, donner des indices.

\subsection{Méthode}

L'analyse prosodique nécessite la mise en place d'un protocole expérimental rigoureux pour l'exploitation et l'interprétation des données Les données sont enregistrées puis transcrites, et une première analyse auditive effectuée.

Elles sont traitées par Praat, logiciel d'analyse de la voix. Le traitement consiste en une numérisation du corpus,

- convertion en .wav des enregistrements audio.

- extraction des fichiers sons pour les séquencer via Praat. Les fichiers numérisés sont ensuite 'découpés' en 2 puis encore en 2 et ainsi de suite pour finir par extraire des extraits homogènes en durée de $6 \mathrm{~s}$ pour ce qui nous concerne.

Enfin, l'étiquetage des signaux de parole est tapuscrit, paramètre par paramètre sur des lignes (les «tiers »), selon les mesures données par le logiciel lorsqu’on déplace un curseur sur l'image. 
Exemple :

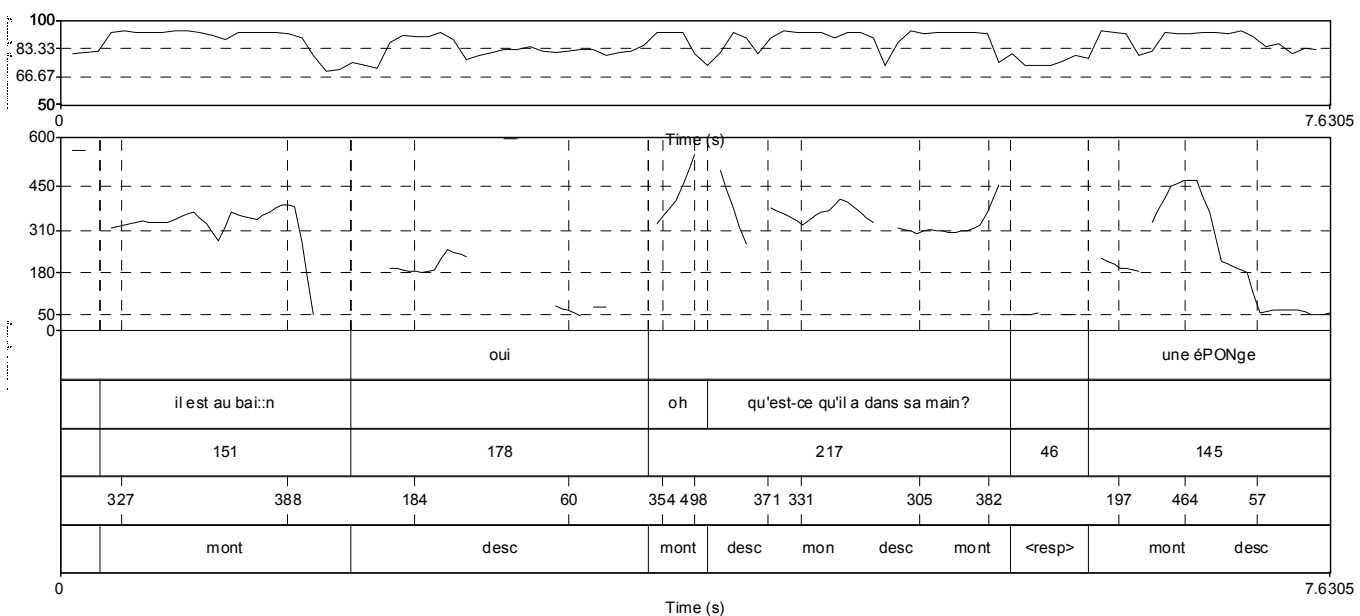

Enfant : il est au bain?

Mère : oui

Enfant : oh! qu'est - ce qu'il a dans la main?

Mère : une éponge

- La courbe supérieure indique l'intensité (limite inférieure paramétrée $=50 \mathrm{~dB}$, limite supérieure $=$ $100 \mathrm{~dB}$ et spectre découpé en trois plages que nous intitulons inférieur, médian, supérieur. Cette notion de niveau est suffisante et nécessaire pour apprécier les variations d'intensité lors d'un même énoncé. Pour le visionnage de la fréquence fondamentale, l'image de la courbe affichée est paramétrée entre $0 \mathrm{~Hz}$ et 600 Hz. Cependant ces valeurs sont relatives et la valeur 0 n'a pas d'intérêt en soi.

- Le second tracé indique les variations de fréquence fondamentale (F0), en 4 niveaux. ( selon la méthodologie de l'Université de Paris 3 - Sorbonne nouvelle, permettant d'identifier les niveaux intonatifs en déterminant le point le plus haut et le point le plus bas et les niveaux intermédiaires).

- L'étiquetage de la courbe est ensuite effectué par paramétrage de cinq lignes : la $\mathbf{1}^{\text {ère }}$ pour coder ce que dit la mère, la $2^{\text {ème }}$ pour coder ce que dit l'enfant, la $3^{\text {ème }}$ pour inscrire les durées des segments (en cs), la $4^{\text {ème }}$ pour coder des points précis de fréquence fondamentale (en $\left.\mathrm{Hz}\right)$, et enfin la $5^{\text {ème }}$ pour marquer les variations prosodiques comme suit $:$ desc $=$ courbe descendante, mont $=$ courbe ascendante, cloche $=$ courbe ascendante /descendante, plat $=$ courbe recto-tono, $\mathrm{P}=$ pause. Si l'on a un seul locuteur, alors l'une des 2 lères lignes n'apparaît pas. Ce codage se fait manuellement, les mesures étant données par Praat

Dans une perspective interactionniste, nous avons analysé le corpus selon un regard croisé portant d'une part sur les conduites langagières dans l'interaction, d'autre part sur l'analyse des paramètres prosodiques.

Une première analyse linéaire intégrale d'un des scenarii met en évidence la variabilité de mouvements prosodiques non prévisibles, co-construits, liés à l'enchaînement des interactions et aux contraintes de l'activité dialogale, qui conduit l'enfant comme l'adulte à une gestion adaptative des échanges. L'utilisation d'un observable plus précis est nécessaire.

De ce fait, nous référant aux fonctions didactiques de l'oral (François 1984, Plane et Garcia-Debanc 2004), nous avons distingué dans chaque scénario :

- les moments de conversation «à bâtons rompus » : le langage est 'moyen expression', et l'activité est conversationnelle.

- les conversations dominées par la transmission d'un contenu : le langage est avant tout pour l'adulte moyen d'enseignement et pour l'enfant objet d'apprentissage. 
- les situations de soliloque pendant lesquelles l'enfant se coupe de la co-énonciation.

Tout en restant dans le cadre pragmatique initial, nous nous plaçons donc dans une perspective microgénétique (Rosenthal 2004).

\section{Résultats}

\subsection{Les situations dialogales spontanées adulte - enfant « à bâtons rompus », en langage en situation ou d'évocation}

On entendra par langage de situation le langage lié à l'action, hic et nunc, et par langage d'évocation le langage pendant lequel l'enfant se décentre du moment présent pour évoquer des évènements passés ou se projeter dans le futur.

- Du point de vue de la forme les interventions sont définies par des critères linguistiques. On note une correspondance syntactico-prosodique. Il n'y a pas d'ambiguïté sur l'interprétation de l'intention de l'enfant par la mère.

- En ce qui concerne la fréquence fondamentale, les courbes présentent de manière générale des patrons mélodiques très variés. La prosodie peut accompagner les formes linguistiques canoniques des actes de langage (Delattre 1966), mais l'enfant manifeste déjà, en construisant le sens, un contenu prosodique implicite qui étoffe le contenu explicite de l'énoncé : à un même acte de langage correspondent des formes linguistiques différentes marquées intonativement, comme chez l'adulte.

- Plus spécifiquement, les courbes des interventions 'complexes' c'est-à-dire qui associant plusieurs actes de langage sont souvent caractérisées par un soulignement contrastif qui marque un focus attentionnel. Ce n'est pas un phénomène de focalisation spécifique de l'enfant mais une co-construction référentielle. Ce soulignement est porté par le dernier acte de langage de l'intervention.

- La durée de chaque intervention est variable. On remarque qu'en langage d'évocation la longueur des interventions 'complexes' est supérieure à celles produites en langage de situation.

Exemple 1:

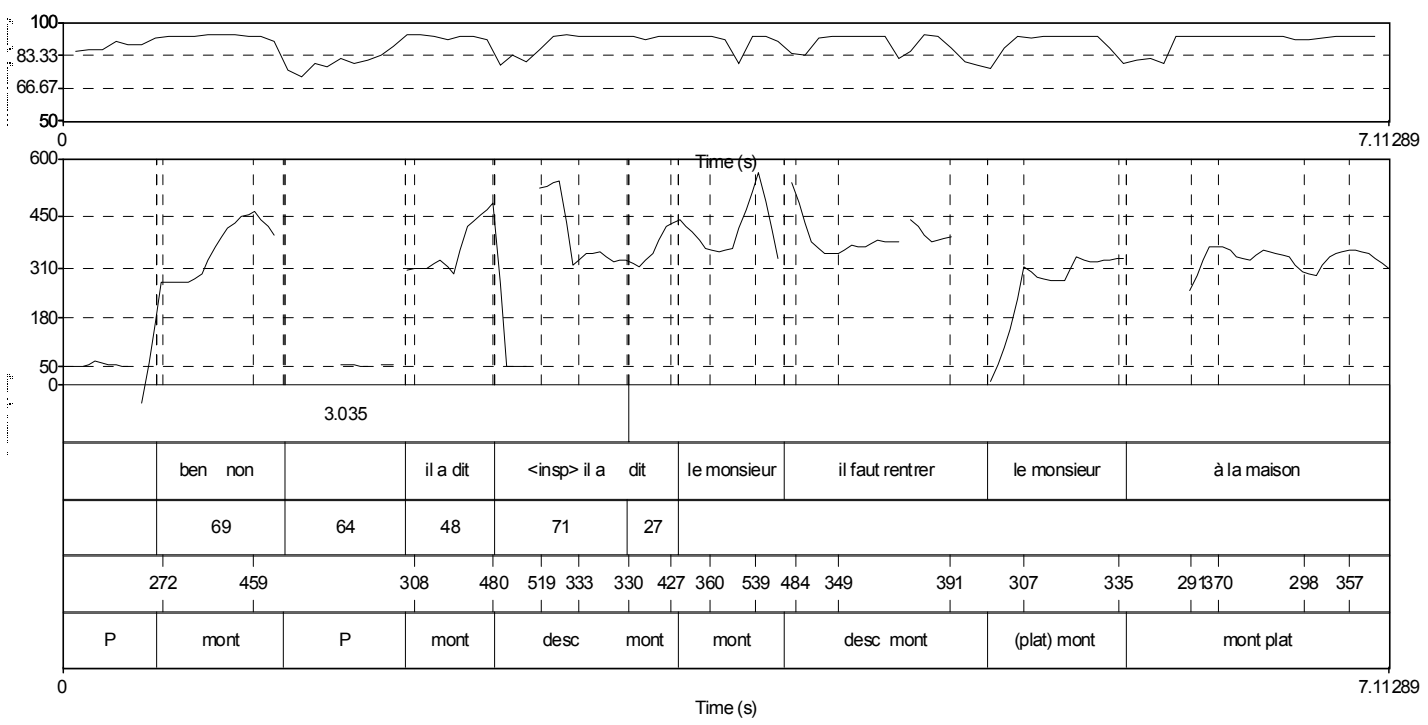

La mère vient de dire : il vous a dit de rester, le monsieur?

Réponse de l'enfant : ben non / il a dit / (inspiration) il a dit le monsieur, il faut rentrer, le monsieur, à la maison.

Analyse de l'exemple : ben non courbe ascendante, modalité exclamative. 
il a dit: montée intonative, coupée par l'inspiration.

il a dit le monsieur : courbe ascendant, soulignement contrastif sur «le monsieur» - il faut rentrer le monsieur à la maison : incise (= rupture vers le bas car reprise) sur le second «le monsieur».

Interprétation: L'analyse de l'ensemble des interactions dans les conversations à bâtons rompus montre que lorsque la centration de l'enfant porte uniquement sur l'activité discursive (langage en situation, langage d'évocation, jeux dialogués interactifs), on se trouve au plan prosodique dans une problématique comparable à celle de l'adulte et l'organisation discursive se marque par divers ordres de balisage. Les indices suprasegmentaux assurent la démarcation et la cohésion des paragraphes de l'oral. Dans notre corpus, le 'paragraphe oral' correspond souvent à l'intervention. On relève la prédominance de l'association assertif - directif en langage en situation et d'expressif - assertif pour le langage d'évocation, l'expressif faisant fonction de préambule et l'assertif de rhème. Ceci se vérifie dans la production d'interventions à acte de langage unique comme dans les interventions 'complexes'.

\subsection{Les conversations dominées par la transmission d'un contenu}

Elles portent :

- d'une part sur la structuration du lexique (période de l'explosion lexicale)

- d'autre part sur des productions textuelles orales qui relèvent de la représentation de l'écrit. Ce sont des situations langagières tout à fait spécifiques, qui, faisant intervenir un médium particulier: le livre, exigent un changement de mode communicationnel.

L'étude instrumentale (cf exemple 2) montre que toute intervention comporte au plan intonatif des traces de l'intention du locuteur, dont l'analyse fournit un schéma au processus interprétatif, et ce, quel que soit le locuteur, mère ou enfant.

Exemple 2 :

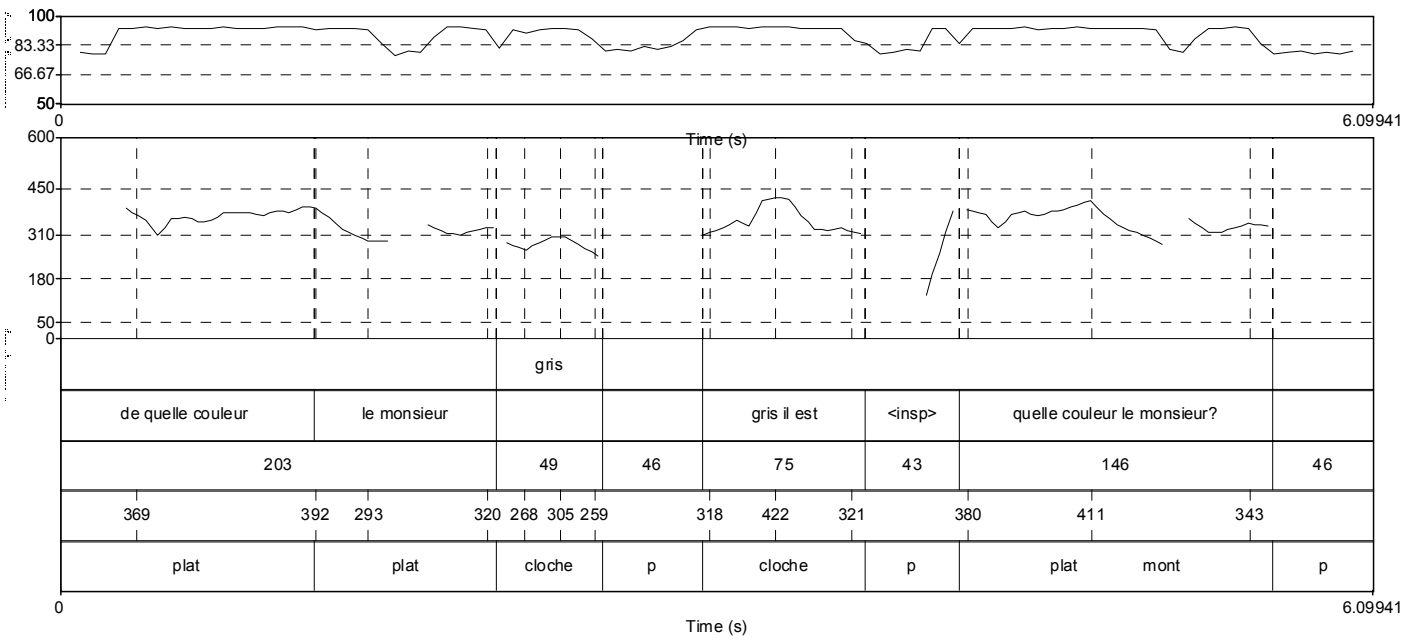

Enfant : il est de quelle couleur le monsieur?

Mère : gris.

/pause/

Enfant : gris il est /inspiration/ il est de quelle couleur le monsieur?

Analyse de l'exemple :

Nous sommes dans une situation didactique au sens large. Les interventions de la mère ont pour but de focaliser l'attention de l'enfant non sur l'activité conversationelle, mais sur le produit du discours, afin 
qu'il effectue un apprentissage précis. On constate que dans ce cas (et cela se vérifie pour toutes les courbes dans ce type de situation), Fo est plate, mais l'intensité est modulée.

Interprétation : Lorsque l'intention de la mère est la centration de l'enfant sur le produit du discours (activité d'apprentissage lexical, syntaxique, concept à formuler), alors on constate chez elle une stratégie prosodique particulière : Fo est plat, l'intensité est modulée. Cela se traduit physiologiquement par une diminution de la pression sous glottique : les variations de Fo sont compensées par un abaissement et une contraction plus forte du larynx, et le fondamental n'est plus modulé. A l'écoute l'effet est compensé par les variations d'intensité. La durée totale des interventions est indépendante de l'utilisation de la stratégie. Cependant on constate que plus l'énoncé est long, plus le segment réalisé sans modulation est ciblé.

Lorsque l'enfant se centre sur le produit du discours, la stratégie utilisée est identique, et ce quelle que soit la nature de l'apprentissage concerné et sa mise en mots.

Il semble donc que lorsque les dialogues mère - enfant ont une fonction transmissive (lexique nominal, référents nominaux, désignation nominale des personnes, apprentissage des couleurs, apprentissage du schéma corporel, lexique verbal et mots grammaticaux), la mère exerce une médiation sémiotique marquée intonativement et aide l'enfant à construire ses apprentissages :

\subsection{Les situations de lecture}

Les situations de lecture sont des situations de production verbale caractérisées par une transposition exigeant un changement de mode communicationnel.

La comparaison des stratégies prosodiques mises en œuvre dans une situation de lecture partagée (centration des locuteurs sur l'activité conversationnelle ou centration sur le produit de l'activité " ce que je dis ») et lors d'un simulacre de lecture (centration de l'enfant sur l'objet même de l'activité « qu'est-ce que je fais quand je lis ?»), montre que l'activité de production verbale orale se différencie notamment en fonction de la nature de la tâche langagière que le jeune enfant montre qu'il veut accomplir.

Exemple 3 : (cas de lecture autonome, « simulacre » de lecture, extrait)

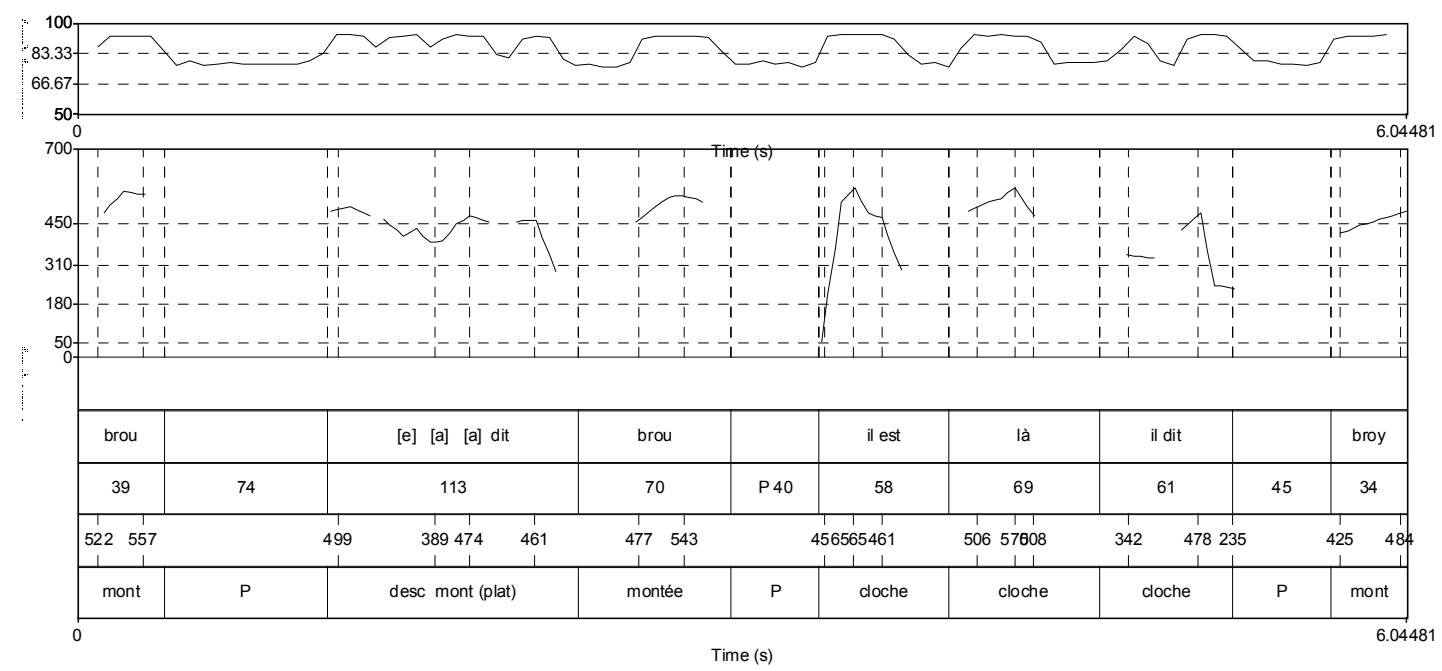

L'enfant est en train de lire, seule, à l'intention de sa grande sœur, un livre : « Mon ami Spot » Enfant : brou / pause/ /e/ /a/ /a/ dit / pause/ il est là il dit / pause / brou 
Analyse de l'exemple : Alors que l'enfant est capable d'un langage articulé compréhensible (qu'il utilise par ailleurs en situation de lecture partagée), certaines de ses interventions ne sont pas compréhensibles à l'écoute: la durée syllabique est variable et non régulière, les pauses ne structurent pas l'énoncé, la fréquence fondaùmentale et l'intensité sont élevées.

Interprétation: Lorsque la centration de l'enfant est sur l'objet même du discours (qu'est-ce que je fais quand je lis ?), il utilise un faisceau de stratégies particulières, prosodiques et verbales : Fo +, I+, absence de pauses structurantes et production orale mixte (associant des segments articulés et des segments en protolangage), durée syllabique variable. Ces productions ne sont pas dirigées.

La prosodie nous renseigne sur la manière dont l'enfant construit ses premières compétences métalinguistiques.

Autrement dit, la production verbale proprement dite met en œuvre une activité de catégorisation implicite de la situation de production, et comporte donc un niveau métadiscursif ou métacomunicationnel

\subsection{Conclusion (1)}

On peut donc conclure que déjà, vers deux ans et demi, les variations de durée, la gestion des pauses et les variations du fondamental permettent de caractériser les 3 niveaux de centration lors du travail de production verbale :

1) la centration porte sur l'activité (la conversation) : les stratégies prosodiques de l'enfant sont celles de l'adulte et on se trouve dans une problématique prosodique identique à toute interaction verbale.

2) la centration porte sur le produit de l'activité (ce qui est dit) : les courbes montrent une absence de modulation de la fréquence fondamentale mais des variations de la courbe de l'intensité. Cette stratégie n'est pas volontaire et est utilisée par la mère comme par l'enfant lorsque la situation est didactique (centration sur l'apprentissage (mot, concept)).

3) et enfin la centration porte sur l'objet de l'activité (ce que l'on fait en disant) : l'enfant utilise un faisceau de startégies particulières qui laisse penser, comme le montrent les tests de perception effectués lors du travail de recherche, à une "régression langagière »: l'adulte ne comprend pas ou mal ce que l'enfant dit, alors qu'il est tout à fait capable de le formuler correctement. On peut en déduire que l'enfant, construisant ses compétences métalinguistiques, se centre sur ce qu'il fait au détriment de ce qu'il dit.

Cette catégorisation implicite et marquée intonativement de la situation de production participe à l'apprentissage.

\subsection{Les situations de soliloques}

Notre corpus ne comprend que $2 \%$ de productions verbales soliloquées, situations pendant lesquelles l'enfant se coupe de la co-énonciation. Ceci est dû aux limites de nos situations d'enregistrement (dyade). Selon la littérature elles sont nombreuses. Piaget constatait déjà qu'il constituait la moitié des productions verbales de l'enfant de 3 ans et le quart à 7 ans

Nous avons répertorié 4 formes de soliloque :

- la répétition 'écholalique', répétition immédiate sans pause en cours d'interaction. L'enfant répète pour lui-même tout ou partie de l'énoncé qui précède.

- la rupture coénonciative : le soliloque est introduit au cours d'une interaction. L'enfant parle en se coupant de la coénonciation. 
- la communication égocentrique, lorsque l'enfant parle seule sans s'adresser à des personnes ou à des objets.

- le jeu d'imitation pendant lequel l'enfant joue un rôle, parle aux objets.

L'analyse prosodique de toutes les interventions soliloquées montre que, si la fonction première du soliloque est l'autorégulation, il n'est pas réductible à un seul type :

1) lors des répétitions 'écholaliques', le segment répété suit toujours sans aucune pause l'énoncé qui précède, et la mère ne le reprend jamais. L'enfant adapte son fondamental à celui de l'interlocuteur dont il répète les propos. Le discours se construit cependant à 2 voix, l'enfant imposant par ses reprises la prise en compte du mélange discursif.

Exemple 1:

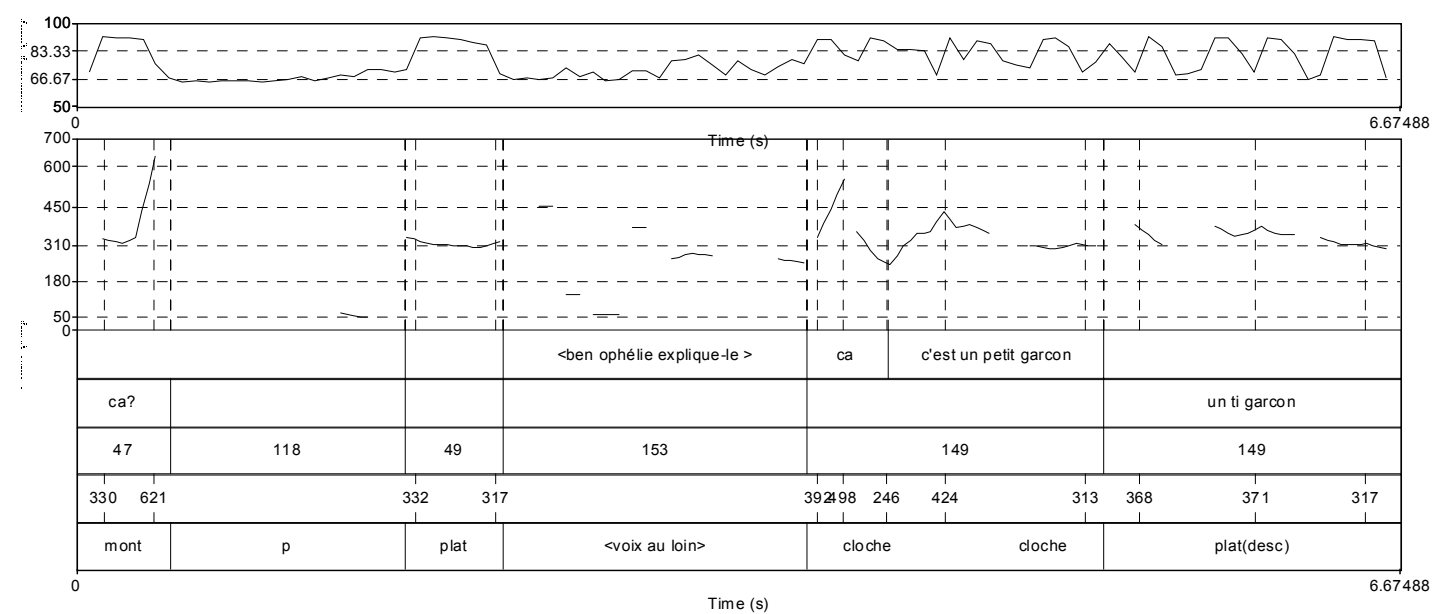

Mère : ben ophélie, explique-le.

Ophélie : ça c'est un petit garçon

Enfant : un ti garçon

2) l'expression des affects est souvent présente notamment par la production d'énoncés chantonnés : registre de Fo plutôt bas, absence de fortes modulations et isochronicité syllabique (autour de $40 \mathrm{cs}$ ). Cette dimension est essentielle mais non spécifique au soliloque.

Exemple 2 : exemple de chantonnement

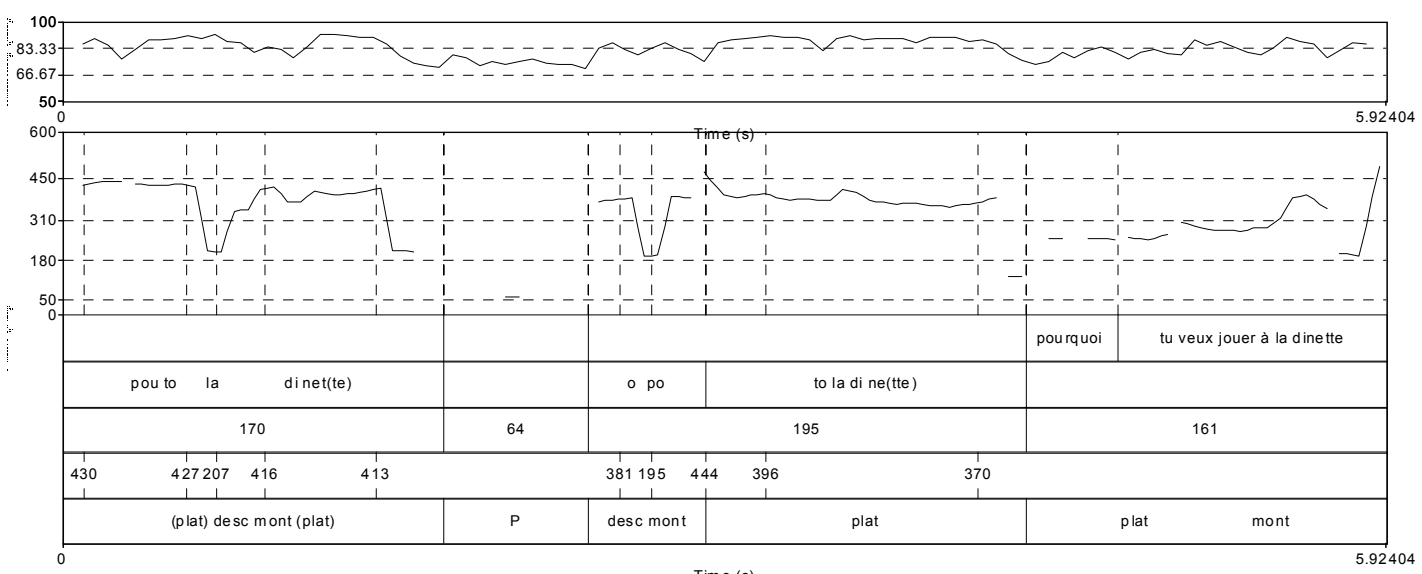


(elle joue avec le couteau de sa dinette)

Enfant : a pou to la dine /141/pouto la dine(tte) /64/ o pouto la dinette

3) lors des énoncés "autodirigés" : rupture coénonciative, communication égocentrique et jeux d'imitation, les stratégies intonatives ne sont pas stables. Les formes prosodiques du soliloque indiquent des degrés de centration de l'enfant différents :
a) lorsque le langage est articulé :

a.1) On retrouve dans l'énoncé soliloqué toutes les caractéristiques prosodiques de l'activité conversationnelle.Les startégies sont variées. On note cependant souvent l'utilisation du registre mélodique haut (N4) qui indique dans ce cas la rupture énonciative avec le locuteur présent. La centration de l'enfant porte sur ce qui se dit, sur l'activité.

a.2) Comme dans l'activité duelle, lorsque l'enfant se centre sur le produit de l'activité (la signification de ce qu'il est en train de dire), sur lequel il focalise son attention car il est en train de s'interroger ou d'apprendre quelque chose de nouveau, il utilise une stratégie prosodique particulière : Fo plat, I modulée. Cette stratégie peut s'accompagner d'un allongement syllabique.

b) lorsque le langage est mixte (ou protolangage) :

L’énoncé soliloqué présente des caractéristiques vocales, rythmiques et mélodiques spécifiques qui traduisent, comme en situation dialogale, la centration de l'enfant sur l'objet même de la tâche cognitivodiscursive à accomplir. Les stratégies prosodiques utilisées ne permettent pas pour l'écouteur la compréhension du message. Les courbes de Fo complexes avec allongements vocaliques aléatoires, les pauses non structurantes de l'énoncé et la longueur des segments confèrent à l'ensemble de l'intervention un mouvement arythmique.

Exemple 3 :

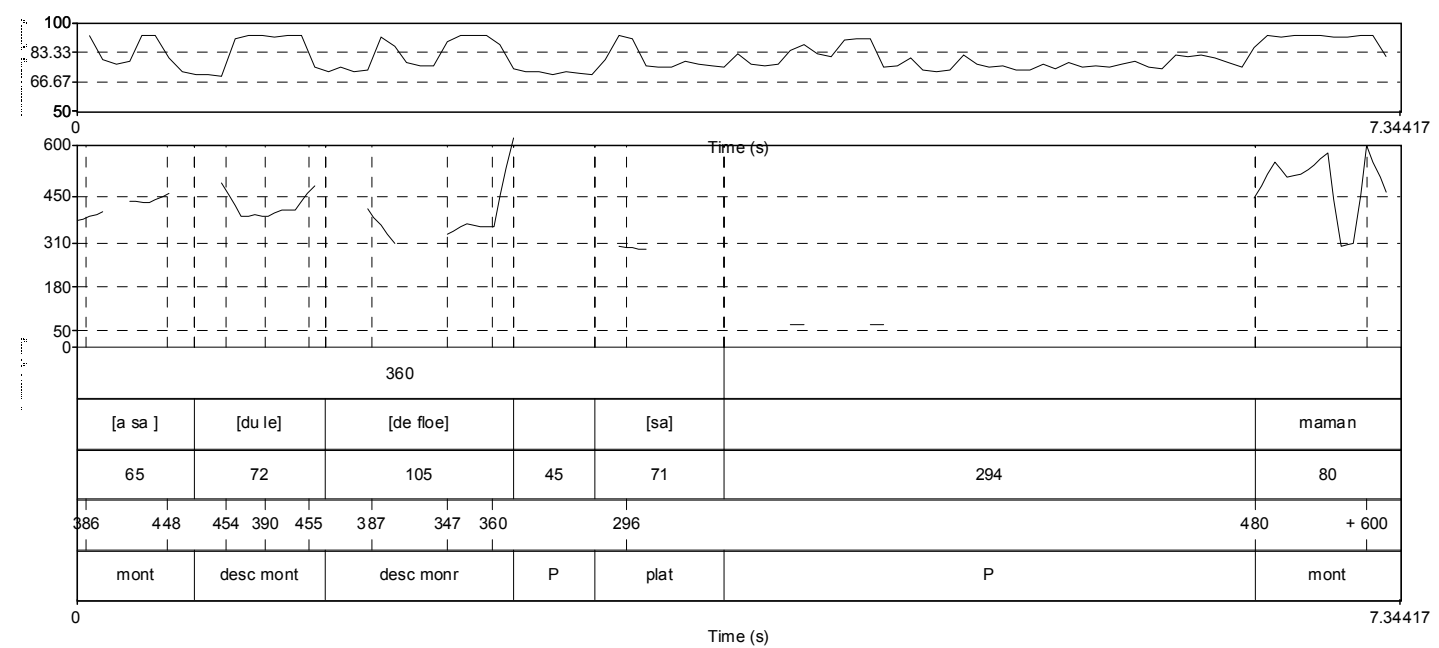

Enfant : [des rouè // --a --a --è --on va ranger le li, on va ranger le li. //<bruits $>$--les --sa --é --le li (bruits)//] -- /asadiledefloe (pause) /sa/ (pause).// maman ?//

\subsection{Conclusion (2)}

Les stratégies prosodiques mises en œuvre par l'enfant en situation de soliloque ne diffèrent pas de celles mises en œuvre en situation de dialogue. 
L'ensemble des tracés suggère que, comme pour les productions langagières en interaction, le soliloque met également en œuvre une activité de catégorisation implicite de la situation de production, selon la nature de la tâche à accomplir (activité conversationnelle / activité d'apprentissage / attitude métalinguistique).

L'analyse prosodique des occurrences soliloquées montre, comme l'avait mentionné Vygotski en son temps, que, lorsque l'enfant est petit, le soliloque ne se distingue du «langage social» qu'essentiellement sous le rapport fonctionnel, et moins au plan de la structure.

\section{Conclusion générale}

L'étude des conditions de production du langage, et notamment la nature de la tâche que l'enfant est en train d'accomplir, nous permet de conclure que la fonction de la prosodie ne se limite pas à la mise en place des relations syntaxiques entre les constituants de l'énoncé et des modalités intersubjectives : elle a une fonction cognitive puisque, dès les premières maîtrises syntaxiques complexes, la production verbale met en œuvre une activité de catégorisation implicite de la situation de production, dans le dialogue comme dans le soliloque.

Quel que soit l'interlocuteur, l'intonation intervient à la manière d'un guidage dans le traitement cognitif de l'information selon un axe double :

- la production d'entités prosodiques spécifiques,

- la perception d'entités prosodiques spécifiques et leur interprétation.

Les diverses stratégies sont inconscientes et varient en fonction de la nature de la tâche que l'un des locuteurs montre qu'il veut accomplir ou faire accomplir.

Le dialogue mère - enfant se réalise dans la collaboration de ces modes de raisonnement, ce qui crée une structure prosodique dynamique, co-construite :

La mère exerce une médiation sémiotique et aide l'enfant à construire ses apprentissages. Cette médiation s'exprime à différents niveaux, et notamment au niveau prosodique.

L'enfant sait utiliser les différentes variables prosodiques qui influencent ses performances :

- il contrôle sa production car il les utilise en fonction de la tâche qu'il veut accomplir.

- il s'adapte aux activités de production ou de réception du discours en fonction des contraintes situationnelles.

- enfin il utilise de manière différenciée les différentes stratégies tant en réception qu'en production.

Concernant donc la question de la stabilisation des structures intonatives ou de la spécificité de stratégies prosodiques chez le jeune enfant, on retiendra deux aspects :

- l'existence de stratégies intonatives spécifiques lorsque l'enfant est centré sur l'objet de l'activité, que l'on ne retrouve pas chez l'adulte, et qui correspondent à une réflexion épilinguistique, aux prémices d'une conscience métalinguistique.

- des stratégies spécifiques en situation didactique, utilisée par l'adulte comme par l'enfant, qui posent le problème de la spécificité de ce type de stratégies : les retrouve-t-on dans les interactions didactiques entre adultes ? Nous travaillons actuellement sur ce type d'interaction.

En tous cas, l'activité d'apprentissage de l'enfant a besoin de toutes les formes de pratiques langagières préparatoires qui conditionnent le travail de formulation. Les différentes stratégies prosodiques sont un fil conducteur dans l'organisation du dialogue, elles participent à la dimension interactionnelle et dialogique des échanges, et leur étude constitue l'un des niveaux d'analyse du dialogue à prendre en compte.

L'intonation est donc un paramètre majeur qui permet une activité de catégorisation implicite de la situation de production et détermine la nature de la tâche à accomplir. Elle a un rôle dialogique, en ce sens qu'elle est une manifestation de ce qui, dans la parole d'un locuteur, dépend de la parole de l'autre : ce qui est dit ne constitue pas le reflet de l'intention du locuteur, mais la manière dont cela est dit s'entend comme 'contre parole' (Baktine 1978). En ce sens, les représentations des interlocuteurs ne correspondent pas uniquement à l'actualisation d'une possibilité inférentielle, mais, au contraire, à des mouvements à travers lesquels ils se positionnent dans l'espace discursif. 
Ceci nous inscrit dans une approche cognitive qui repose, comme le souligne Lacheret-Dujour (2007), sur « une version gestaltiste et constructionniste des formes prosodiques et de leur valeur sémantique, sur l'importance accordée à l'observation et à la modélisation pour le développement de représentations formelles cognitivement pertinentes, et sur la dimension majeure des faits de perception ». D'une manière plus générale, ces observations nous permettent considérer la prosodie comme l'une des composantes langagières qui permet la configuration de champs perceptuels formant la base de co-représentations, dynamiques, configurations à un moment donné, participant à la construction de l'espace interdiscursif.

\section{Références bibliographiques}

Bakhtine M. Esthétique et théorie du roman, Paris, NRF, Gallimard (trad française D.Olivier)

Bassano D., Maillochon I., Eme P. (1998) "Developmental changes and variability in early lexicon: a study of French children's naturalistic productions", Journal of Child Language, 25, pp 193-531.

Bernicot J. (1992) Les actes de langage chez l'enfant, Paris ; PUF.

Bourhis V., (2005) Contribution à l'étude de la prosodie du français parlé chez l'enfant entre 2 ;6 et 3 ;1.Etude de cas, Thèse de doctorat, document non publié.

Briand (1998) "Feature integration and spatial attention : More evidence of a dissociation between endogenous and exogenous orienting", Journal of experimental psychology. Human perception and performance, 24, n' 4 , pp. $1243-1256$

Candea M. (2000) Contribution à l'étude des pauses silencieuses et de phénomènes dits d'hésitation en français oral spontané, Thèse de doctorat, Université Paris 3 (document non publié)

Cohen S. (1978) "Environmental load and ths allocation of attention, in A.Baum, J.E.Singer et S. Valins (Eds), Advances in environmental psychology, vol 11, pp 1-29, N.J : Lawrence Erlbaum

Crystal D. (1969) Prosodic systems and intonation in English, Londres, Cambridge, University Press.

Delattre P. (1966), "Les dix intonations de base du français", French Review, 40 (1), Illinois, American Association of teachers of french, pp 1-14.

Dore J. (1975) "Holophrases, speech acts, and language universals", Journal of Child Language, 2, pp21-40.

Fongagy I. (1983) La vive voix. Essai de psychophonétique. Paris, Payot.

Francois F., Hudelot C., Sabeau-Jouanet S. (1984) Conduites linguistiques chez le jeune enfant, PUF.

Fuchs C., Le Goffic, (1985) Initiations aux problèmes des linguistiques contemporaines, Paris, Hachette.

Gerard C., Clement J., (1998) "The structure and development of French prosodic representations", Language and Speech, 41 (2), pp 117-142.

Halliday M.A.K. (1975) Learning how to mean - exploration in the development of language, Londres, Arnold.

Konopczynski G. (1990) Le langage émergent : caractéristiques rythmiques. Hambourg, Helmut Buske Verlag.

Konopczynski G. (1991) Le langage émergent II . Aspects vocaux et mélodiques. Hambourg, Helmut Buske Verlag.

Konopczynski G. (2000) "The development interactive intonology model : applications to French", Parole, vol 7/8, pp 177-201.

Lacheret-Dujour A., Beaugendre F. (2002) La prosodie du français, CNRS Editions.

Lacheret-Dujour A. (2007) «Une interface à multiples facettes », in Interfaces Discours-Prosodie, Nouveaux cahiers de lingusitique, 28 , Université de Genève, p 7-40

Martin P. (1981), "Pour une théorie de l'intonation. L'intonation est-elle une structure congruente à la syntaxe ?", in M. Rossi (es) : L'intonation : de l'accent à la sémantique. Paris : Klincksiek.

Morel M.-A., Danon-Boileau L. (1992), La deixis, Paris, PUF. 
Morel M.A., Danon Boileau L. (1998) Grammaire de l'intonation : l'exemple du français, Faits de Langue, Ophrys.

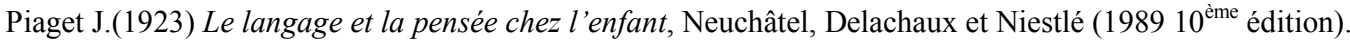

Plane S. (2004) "L'enseignement de l'oral : enjeux et évolution " in Comment enseigner l'oral à l'école primaire (coord C. Carcia-Debanc, S. Plane) Hatier.

Rosenthal V. (2004) L'anticipation à l'horizon du présent. R.Sock et B. Vaxelaire Eds Liège : Mardaga.

Rossi M. (1999) L'intonation, le système du français : description et modalisation. Paris, Ophrys.

Rossi M., Di Cristo A., Hirst D., Martin P., ET Nishinuma Y. (1981) "L’intonation : de l'acoustique à la sémantique", Etudes Linguistiques XXV, CNRS ; Institut de phonétique d'Aix-en-Provence, Paris : Klincksiek.

Searle J.R., Vanderveken D. (1985) Foundations of illocutionary logic, Cambridge, Cambrigde University press.

Vroomen J., Collier R., Mozziconacci S. (1993) "Duration and intonation in emotional speech", Proceeding of Eurospeech 93, $3^{\text {rd }}$ european conference on speech communication and technology, vol 1, Berlin, Germany.

Vygotski LS. (1934) Thought and language, Moscou, Stosekgiz., Traduction anglaise : Cambridge, M.I.T. Press, 1962.Traduction française : Pensée et langage, Paris, La dispute/ SNEDIT, $3^{\text {ème }}$ édition, 1997. 